\title{
A Note on the Globular Forms of Vibrio cholerae
}

\author{
By THE LATE P. BRUCE WHITE \\ National Institute for Medical Research, Hampstead, London
}

SUMMARY: The cultivation of Vibrio cholerae in media containing sublethal concentrations of penicillin apparently favours the selection of two morphological forms: motile spherical masses containing large numbers of apparently nuclear elements, and fantastically branched forms. On penicillin-free media both forms yield the original comma forms.

Globular forms of Vibrio cholerae appear in stock cultures in the usual laboratory media, though not in $24 \mathrm{hr}$. cultures of strains freshly isolated from man. They may be produced in large quantities by adding about a quarter of the growth on a $24 \mathrm{hr}$. nutrient agar slope culture of $V$. cholerae to a solution of penicillin containing 25-100 u./ml. Upon incubation, mass growth takes place. The number of viable individuals does not increase, but each vibrio becomes a sphere, which may be up to 8-10 $\mu$ in diameter. The spheres are flagellated and motile, and remain so for several days in penicillin-vibrio mixtures held at $10^{\circ}$. Prolonged incubation of the mixtures produces larger (Pl. 1, figs. 1, 2) spheres up to $12 \mu$ in diameter, which lose motility and staining properties, become 'vacuolated' and may bulge in subsidiary masses from the periphery. It is highly improbable that these spherical forms owe their motility to any form of torque, and it can only be concluded that the stainable flagella on their surfaces are indeed the cause of their locomotion.

By the differential congo-red methylene-blue method (White, 1947) the larger spheres commonly stain blue, and the remainder take up the methylene-blue only when they are dead. After extraction of the spheres to remove nucleic acids, staining with methylene-blue reveals large numbers of granules, which may represent the unit organisms in the vibrionic mass. The spheres have serological properties similar to the parent culture, whether these are Inaba or Ogawa in type.

The penicillin is neither lethal nor particularly injurious and appears simply to suppress directional growth and the usual mode of division, so that unoriented cell growth occurs, without increase in the number of viable individuals in the culture.

Under optimum conditions extraordinary starfish-like forms occur, with tapering branches; many of these may be motile. On staining, the broader part of the branches is seen to consist of double or multiple chains of nuclear elements; the finer branches are formed by simple vibrios, and from these the culture may regenerate, either in its original form, or, if seeded on to fresh penicillin-agar, in the spherical form.

I have considered the globular form as a step in the direction of the vesicular forms that occur in the streptobacilli, but have found no good evidence that this is the case. Another feature of general interest attaches to the branching forms derived from the multinuclear cells that occur both naturally and under 
Journal of General Microbiology, Vol. 4, No. 1

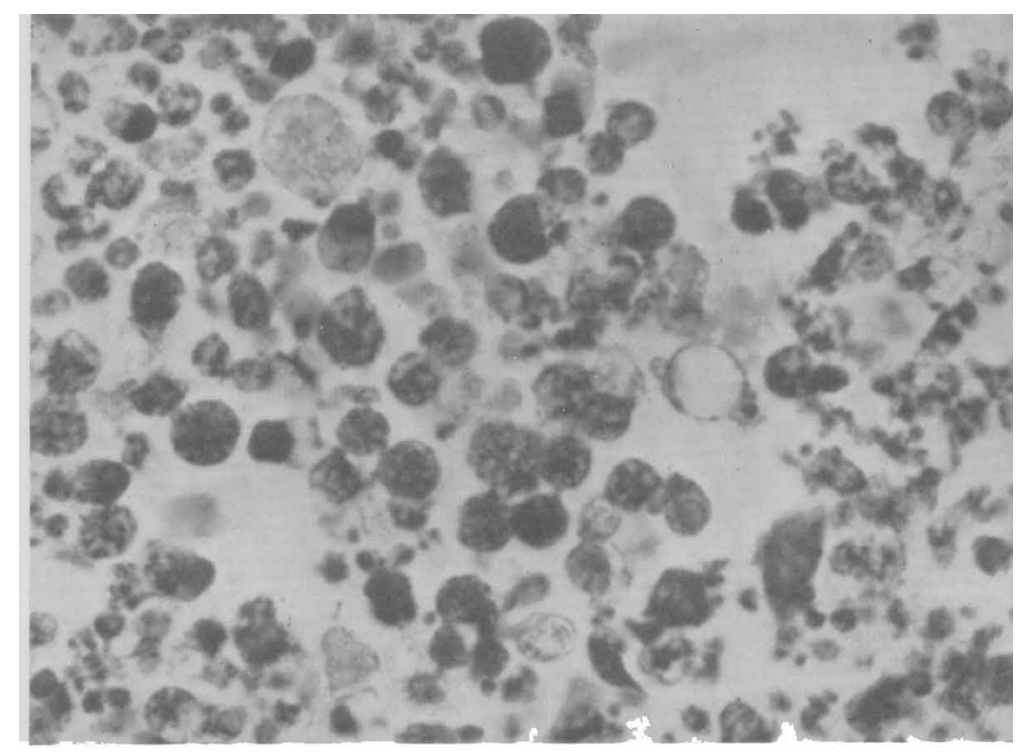

Fig. 1

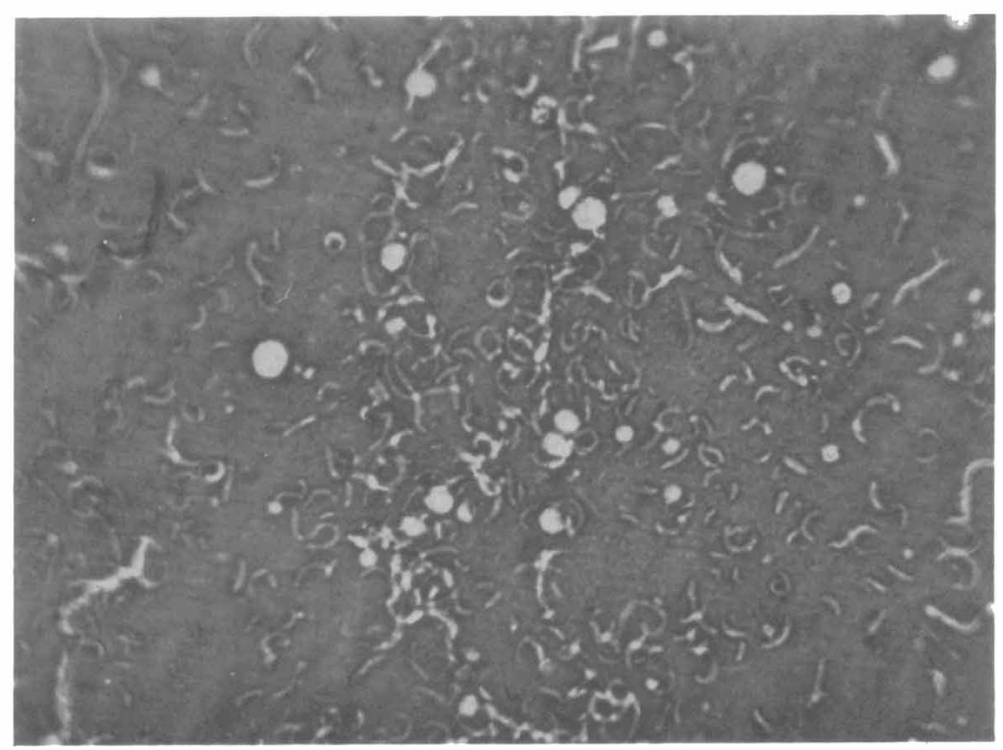

Fig. 2

P. Bruce White-Globular forms of $V$. cholerae. Plate 1 
the influence of penicillin. It is possible that the development of multinuclearity in this way may have been a stage in the evolution of the multinucleate and branched species of bacteria.

\section{REFERENCE}

WhIte, P. B. (1947). A method for combined positive and negative staining of bacteria. J. Path. Bact. 59, 384.

\section{EXPLANATION OF PLATE}

Fig. 1. Globular forms of $V$. cholerae, stained by methylene-blue. $\times 1250$.

Fig. 2. Vibrionic, branched and globular forms of $V$. cholerae. Unstained preparation with a background of nigrosin. $\times 1250$.

(Received 7 April 1949) 